\title{
Total Eclipse of FDG-PET Scan Caused by Hyperphysiological FDG Uptake of Masticatory Muscles Due to Chewing
}

Kursat Okuyucu, Huseyin San, Alper O. Karacalioglu, Ozdes Emer and Semra Ince*

Departments of Nuclear Medicine, Gulhane Military Medical Academy and School of Medicine, Etlik, Ankara, Turkey

"Corresponding author: Semra Ince, M.D., Departments of Nuclear Medicine, Gulhane Military Medical Academy and School of Medicine, Etlik, Ankara, Turkey, Tel: (90) 312 3044828; Fax: (90) 312 3044800; E-mail: since@gata.edu.tr

Received date: Oct 09, 2015, Accepted date: Nov 03, 2015, Publication date: Nov 09, 2015

Copyright: ( 2015 Okuyucu K, et al. This is an open-access article distributed under the terms of the Creative Commons Attribution License; which permits unrestricted use; distribution; and reproduction in any medium; provided the original author and source are credited.

\begin{abstract}
In routine oncological $18 \mathrm{~F}$-fluorodeoxyglucose positron emission tomography (FDG-PET/CT) scan, the patients rest in a quite, dimly lit room for 60 minutes after intravenous (IV) injection of FDG and they are instructed not to move during this time (uptake phase) before FDG-PET/CT scanning to prevent and decrease unwanted FDG accumulations on muscle traces caused by muscle movements. In conditions of patient inconsistency, undesirable activities can be seen and reach high levels making difficult or even impossible to interpret the images. Different muscle groups form various physiological or over- physiological uptake patterns depending on their specific motion. Herein we represent a good didactic example to this phenomenon.
\end{abstract}

Keywords: Masticatory muscles; Physiological muscle activity; 18 fluro deoxy-glucose positron emission tomography (FDG-PET)

\section{Introduction}

Four muscles implement chewing action (mastication) mainly and these are called masticatory muscles. Musculus massetericus, musculus temporalis (pars anterius, pars medius, pars posterius) and musculus pterygoideus medialis (internus) close temporomandibular joint (mouth), while musculus pterygoideus lateralis (externus) opens it [1].

Contracting muscle cells consume much more glucose than they do while resting. They spend the energy after degradation of glucose for their increased work load and locomotion. So they take up glucose and its metabolic analogue, FDG in larger amounts during exercise [2]. This condition shows itself as increased physiological or over physiological FDG accumulation along muscle tracing on FDG-PET images [3].

After IV injection of FDG, the patients were instructed to rest lying motionlessly in an obscure room for 60 minutes before FDG-PET/CT scanning to prevent and at least decrease unwanted physiological FDG accumulations making difficult the evaluation of images. If the patients do not comply with these warnings, these activities increase and create problems for interpretation. Different muscles show peculiar physiological uptake patterns relating to their movement [4].This case has been introduced to show dense muscle activity caused by this kind of muscle activation leading to uninterpretable FDG-PET/CT test.

\section{Patient Findings}

55 year-old male diagnosed as lung cancer underwent right upper pulmonary lobectomy followed by radiotherapy. As the patient had shoulder and back pain during the follow-up, a bone scintigraphy was performed and suspicious findings were observed for bone metastasis. Upon this, FDG-PET/CT imaging was requested for restaging in order to reveal possible bone metastasis.
Meanwhile, the patient having complaint of dry mouth for a long time has been recommended to chew sugar-free gum after radiotherapy period by his physician to avoid side effects of radiotherapy on salivary glands resulting in dry mouth because of decreased salivation caused by radiation damage. For this fear, the patient insisted on chewing while he was resting during the uptake phase after IV FDG administration despite all routine warnings told by nuclear medicine technician before the injection. Prominently marked diffuse bilateral uptake in neck conforming the traces of temporal, masseter and pterygoid muscles consistent with over physiological muscle activity due to perpetual chewing action was seen on FDG PET/CT images (Figure 1). This abnormal muscle accumulation caused by chewing steadly rendered impossible to interpret the images by storing a great tangible portion of the injected activity on its own account and the test was cancelled. A new scan scheduled several days later in which the patient did not chew resulted in normal physiological FDG distribution and the test was completed (Figure 2).

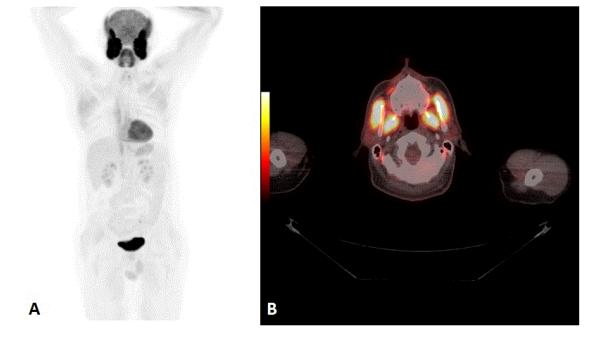

Figure 1: A. Maximum intensity projection (MIP) image and B. axial slice of FDG PET/CT scan showing excessive FDG acummulation of bilateral temporal, masseter and pterygoid muscles, equal to the bladder, but greater than brain and heart uptake. 


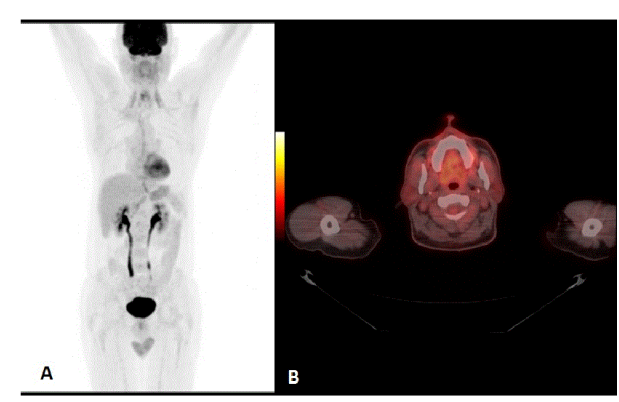

Figure 2: A. Maximum intensity projection (MIP) image and B. axial slice of repeated FDG PET/CT scan. Normal physiologic FDG distribution is seen.

\section{Discussion}

Muscle uptake of FDG is a cause of false-positivity on PET images [5]. On all FDG-PET/CT images, physiological muscle uptakes are seen in a certain degree depending on patient's resting status. It is not possible to eliminate these physiological accumulations completely, but possible to hold them in tolerable levels warning the patient to rest fully [6]. In spite of all the warnings, the patients may disobey and some physiological muscle accumulations come out in varying amounts.

Exercising muscles utilize much more glucose than their resting status. They spend the metabolic energy of glucose degradation for their increased work load. For this purpose, they take up glucose into myocytes and metabolize it for energy production. FDG is a metabolic analogue of glucose and is also taken up by myocytes, but FDG does not go in metabolic pathways further and stays trapped in muscle cells. This condition shows itself as increased physiological or over physiological FDG accumulation along muscle tracing on FDG-PET images [3].

Common areas of muscle uptake are head and neck region, thorax, upper extremities; less commonly lower extremities and diffuse whole body [7]. Special muscle groups form peculiar uptake patterns depending on their anatomical location and motion. Uptake of laryngeal muscles taking part in speech formation was represented as a result of talking during the uptake phase [8]. Intense symmetric activity in the masseter and temporal muscles associated with gum chewing was shown [9]. Anxiety may cause accumulation in neck muscles [5]. Scalene muscle activity may be seen as a physiological variant [10]. Increased uptake in sternocleidomastoid muscle owing to straining the neck, deep breathing or intense coughing [7]. Thoracic or abdominal muscles (diaphragma, intercostal muscles) may be visible in patients with hard breathing or coughing secondary to chronic obstructive pulmonary disease. The flexor/extensor carpi and digitorum muscles of the hand would show increased uptake due to writing. Muscles of the lower extremities in anterior compartment (tibialis anterior, extensor digitorum longus, extensor hallucis longus) were the most frequently visualized ones attributed to activities as walking, tapping feet [7]. Diffuse whole body muscle uptake can be the result of insulin intake, strenuous exercise involving many muscle groups.
Pathologic FDG muscle uptakes may be seen in infectious and inflammatory (like polymyositis, dermatomyositis) diseases affecting muscles, benign or malign (rhabdomyosarcoma) muscle neoplasms and trauma [11]. These uptakes occur by a different mechanism than physiological ones. They tend to be more localized and are associated directly with the lesion sites affected by the disease itself.

All these physiological or over physiological muscle activities may sometimes cause confusions while evaluating FDG-PET/CT scan. As they take up most of the injected FDG, they can blur the images or mask visualization of real lesions diminishing their accumulation. Rarely they reach so many high levels that they render impossible to interpret the images as is in our case. In our case, the patient chewed gum during the uptake phase because of dry mouth fear without our information. This led to unusual intense bilateral FDG accumulation over masticatory muscles resulting in the obligation of a repeated test.

\section{Conclusion}

It is not enough to warn the patient superficially to avoid or diminish physiological or over physiological muscle activities by just resting them. Rarely a skipped underlying special or personal condition may exist and eventuate to misinterpretation or termination of the test. So patients must be warned clearly and even watched especially in a few selected cases if possible. A detailed patient history elaborating probable private conditions should be taken.

\section{References}

1. Ma L, Zhou Y, Zhang Y, Xia H, Zhou X, et al. (2015) Biomechanical Effects of Masticatory Muscles on Human Mandible After Reconstructed Mandibulectomy Tumor. J Craniofac Surg .

2. Barrington SF, Maisey MN (1996) Skeletal muscle uptake of fluorine-18FDG: effect of oral diazepam. J Nucl Med 37: 1127-1129.

3. Nakamura S, Okochi K, Murata Y, Shibuya H, Kurabayashi T (2009) [18F]Fluorodeoxyglucose-PET/CT differentiation between physiological and pathological accumulations in head and neck. Nucl Med Commun 30: 498-503.

4. Yüksel M, Eziddin S, Strunk H, Biersack HJ (2006) Contribution of $\mathrm{PET} / \mathrm{CT}$ for differentiation of FDG uptake in lateral pterygoid muscle in a patient with malignant melanoma. Rev Esp Med Nucl 25: 115-116.

5. Shreve PD, Anzai Y, Wahl RL (1999) Pitfalls in oncologic diagnosis with FDG PET imaging: physiologic and benign variants. Radiographics 19: 61-77.

6. Cook GJ, Fogelman I, Maisey MN (1996) Normal physiological and benign pathological variants of 18-fluoro-2-deoxyglucose positronemission tomography scanning: potential for error in interpretation. Semin Nucl Med 26: 308-314.

7. Jackson RS, Schlarman TC, Hubble WL, Osman MM (2006) Prevalence and patterns of physiologic muscle uptake detected with whole-body $18 \mathrm{~F}$ FDG PET. J Nucl Med Technol 34: 29-33.

8. Kostakoglu L, Wong JC, Barrington SF, Cronin BF, Dynes AM, et al. (1996) Speech-related visualization of laryngeal muscles with fluorine-18FDG. J Nucl Med 37: 1771-1773.

9. Case of the month: Elvis sign (2007) Nuclear Pharmacy e-newsletter.

10. Jacene HA, Goudarzi B, Wahl RL (2008) Scalene muscle uptake: a potential pitfall in head and neck PET/CT. Eur J Nucl Med Mol Imaging 35: 89-94.

11. Tateyama M, Fujihara K, Misu T, Arai A, Kaneta T, et al. (2015) Clinical values of FDG PET in polymyositis and dermatomyositis syndromes: imaging of skeletal muscle inflammation. BMJ Open 5: e006763. 